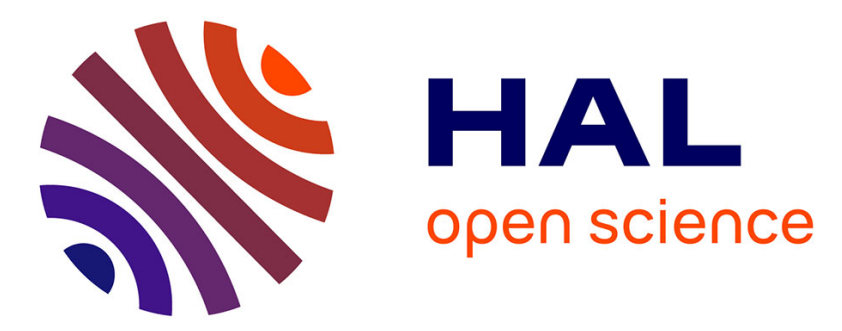

\title{
Peamacléine Pru p 7 : épidémiologie, implications cliniques et place dans le diagnostic moléculaire de l'allergie à la pêche en région méditerranéenne
}

C. Klingebiel, A. Poisson, J. Lidholm, A. Ehrenberg, J. Ostling, V. Liabeuf, C. Agabriel, J. Birnbaum, F. Porri, R. Arif-Lusson, et al.

\section{To cite this version:}

C. Klingebiel, A. Poisson, J. Lidholm, A. Ehrenberg, J. Ostling, et al.. Peamacléine Pru p 7 : épidémiologie, implications cliniques et place dans le diagnostic moléculaire de l'allergie à la pêche en région méditerranéenne. Revue francaise d'allergologie, 2018, 58 (1), pp.16-22. 10.1016/j.reval.2017.07.005 . hal-01791653

\section{HAL Id: hal-01791653 \\ https://hal.science/hal-01791653}

Submitted on 15 Apr 2019

HAL is a multi-disciplinary open access archive for the deposit and dissemination of scientific research documents, whether they are published or not. The documents may come from teaching and research institutions in France or abroad, or from public or private research centers.
L'archive ouverte pluridisciplinaire HAL, est destinée au dépôt et à la diffusion de documents scientifiques de niveau recherche, publiés ou non, émanant des établissements d'enseignement et de recherche français ou étrangers, des laboratoires publics ou privés. 
Article original

\title{
Peamacléine Pru p 7 : épidémiologie, implications cliniques et place dans le diagnostic moléculaire de l'allergie à la pêche en région méditerranéenne
}

\section{Peamaclein Pru p 7: Epidemiology, clinical implications and place in the molecular diagnosis of peach allergy in the Mediterranean region of France}

\author{
C. Klingebiel ${ }^{\mathrm{a}, *}$, A. Poisson ${ }^{\mathrm{b}}$, J. Lidholm ${ }^{\mathrm{c}}$, A. Ehrenberg ${ }^{\mathrm{c}}$, J. Östling ${ }^{\mathrm{c}}$, V. Liabeuf ${ }^{\mathrm{d}}$, C. $_{\text {Agabriel }}^{\mathrm{e}}$, \\ J. Birnbaum ${ }^{\text {d }}$, F. Porri ${ }^{\text {b }}$, R. Arif-Lusson ${ }^{\mathrm{f}}$, M. Gouitaa ${ }^{\mathrm{g}}$, A. Aferiat-Derome ${ }^{\mathrm{h}}$, D. Charpin ${ }^{\mathrm{g}}$, \\ T. Sofalvi ${ }^{\mathrm{g}}$, I. Cabon-Boudard ${ }^{1}$, Y.-P. Massabie-Bouchat ${ }^{1}$, C. Soler ${ }^{\mathrm{a}}$, I. Cleach ${ }^{\mathrm{j}}$, \\ J.-L. Mège ${ }^{\mathrm{j}, \mathrm{k}}$, J. Vitte ${ }^{\mathrm{f}, \mathrm{j}}$, le réseau ANAFORCAL Provence \\ a Laboratoire Montgrand, LBM multisite SELDAIX, BIOPLUS, 13006 Marseille, France \\ b Service de pneumo-allergologie, hôpital Saint-Joseph, 13008 Marseille, France \\ ${ }^{\mathrm{c}}$ Thermo Fisher Scientific, Uppsala, Suède \\ d Service de dermatologie-vénéréologie, hôpital Timone, Aix-Marseille université, AP-HM, 13005 Marseille, France \\ e Service de pédiatrie multidisciplinaire, hôpital Timone, Aix-Marseille université, AP-HM, 13005 Marseille, France \\ ${ }^{\mathrm{f}}$ Inserm UMR 1067 CNRS 7333, Aix-Marseille université, 13009 Marseille, France \\ g Service de pneumologie, hôpital nord, Aix-Marseille université, AP-HM, 13016 Marseille, France \\ ${ }^{\mathrm{h}}$ Cabinet médical, les jardins de Castellane, 45, avenue Jules-Cantini, 13006 Marseille, France

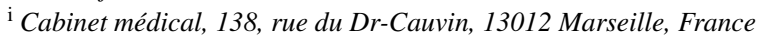 \\ ${ }^{\mathrm{j}}$ Laboratoire d'immunologie, hôpital de la conception, Aix-Marseille université, AP-HM, 13005 Marseille, France \\ ${ }^{\mathrm{k}}$ URMITE, UMR CNRS 6236, 13005 Marseille, France
}

Reçu le 7 juin 2017 ; accepté le 11 juillet 2017

Disponible sur Internet le $1^{\text {er }}$ septembre 2017

\section{Résumé}

Certaines allergies immédiates à la pêche ne sont pas élucidées sur le plan moléculaire par l'exploration des IgE spécifiques utilisées en routine : extrait de pêche, Pru p 1 (PR-10), Pru p 3 (protéine de transfert lipidique), Pru p 4 (profiline) et groupements glucidiques à réactivité croisée, et ce malgré des tests cutanés fortement positifs et une histoire clinique convaincante. L'exploration de ces patients par un test d'activation des basophiles en présence d'extrait de pêche est fortement positive, suggérant l'implication d'un allergène moléculaire de la pêche, absent des outils diagnostiques in vitro actuels. Nous rapportons ici la forte prévalence de la sensibilisation vis-à-vis de la peamacléine (Pru p 7) chez les patients méditerranéens allergiques à la pêche (région de Marseille), ainsi que les caractères épidémiologiques, cliniques et biologiques associés à ce nouvel allergène.

(C) 2017 Publié par Elsevier Masson SAS.

Mots clés : Peamacléine ; Pru p 7 ; Allergie à la pêche ; Allergène moléculaire ; IgE ; Anaphylaxie à la pêche ; Anaphylaxie idiopathique ; Profil moléculaire de la pêche ; Allergène majeur de la pêche

\footnotetext{
Abstract

* Auteur correspondant.

Adresse e-mail : caro.klingebiel@orange.fr (C. Klingebiel).
}

Molecular-based allergy diagnostic kits using standard IgE reagents such as peach extract, Pru p 1 (PR-10), Pru p 3 (non-specific lipid transfer protein), Pru p 4 (profilin), and cross-reactive carbohydrate determinants, are unable to identify the offending allergen in certain patients with peach 
allergy in Southern France, despite positive skin tests and a convincing clinical history. The constant and high positivity of the basophil activation test with peach extract in such patients suggests the involvement of a molecular peach allergen not included in existing in vitro diagnostic kits. Herein we report that peamaclein (Pru p 7) is a major allergen in peach-allergic patients in the Mediterranean (Marseille) region and we describe the epidemiological, clinical and laboratory characteristics associated with this novel allergen.

(C) 2017 Published by Elsevier Masson SAS.

Keywords: Peamaclein; Pru p 7; Peach allergy; Molecular allergen; IgE; Peach anaphylaxis; Idiopathic anaphylaxis; Molecular profile of peach; Major allergen of peach

\section{Introduction}

En fonction de la voie de sensibilisation, l'hypersensibilité immédiate alimentaire peut résulter d'une réactivité vis-àvis de deux types d'allergènes. Les allergènes de classe I sont des allergènes alimentaires responsables d'une sensibilisation primaire au niveau du tractus digestif. Les allergènes de classe II sont responsables d'une sensibilisation secondaire par réactivité croisée d'une sensibilisation primaire via le tractus respiratoire vis-à-vis d'allergènes polliniques homologues. À la fin de la petite enfance et jusqu'à l'âge adulte, l'allergie alimentaire par réactivité croisée est prédominante $(60 \%)$ [1-3].

En Europe centrale et du Nord, l'allergène de classe II le plus fréquent appartient à la famille des PR-10 (Bet v 1 et homologues, dont Pru p 1), moins fréquemment aux profilines (Bet v 2 et homologues, dont Pru p 4). Dans les régions méditerranéennes, il existe une plus forte sensibilisation vis-à-vis des allergènes de la famille des profilines et des protéines de transfert lipidique (LTP). L'allergène Pru $\mathrm{p} 3$ est le chef de file du groupe des LTP « alimentaires et polliniques à réactivité croisée », qui comprend entre autres Mal d 3 (pomme), Pla a 3 (platane), Ole e 7 (olivier) et Art v 3 (armoise). Les prévalences de ces sensibilisations sont corrélées aux prévalences des sensibilisations primaires vis-à-vis des pollens [3,4] .

L'allergie alimentaire aux fruits est une cause fréquente de consultation d'allergologie. Les fruits les plus souvent en cause appartiennent à la famille des Rosacées [4]. Cette allergie alimentaire est le plus souvent due à un syndrome pollen-fruit : pollinose primaire avec sensibilisation secondaire aux fruits. Dans ce cadre, l'allergie alimentaire à la pêche est souvent décrite par un syndrome oral (Pru p 1, plus rarement Pru p 4) et dans de plus rares cas une anaphylaxie sévère (impliquant classiquement Pru p 3).

Dans les régions méditerranéennes, de nombreux cas d'allergie avérée à la pêche ne sont pas élucidés sur le plan moléculaire par la recherche d'IgE spécifiques de la pêche Pru $\mathrm{p} 1$, Pru p 3, Pru p 4 et groupements glucidiques végétaux à réactivité croisée (CCD) [5]. Il existe une discordance entre des tests cutanés (TC) fortement positifs et des valeurs d'IgE contre la pêche très basses, voire indétectables. Le test d'activation des basophiles (TAB) avec l'extrait de pêche et Pru p 3 s'avère positif chez ces patients, suggérant l'implication d'un nouvel allergène [6-8]. En effet, le réactif utilisé pour le TAB étant un allergène moléculaire naturel nPru $\mathrm{p} 3$, une éventuelle copurification de celui-ci avec la peamacléine (Pru p 7) est possible
$[9,10]$. Nous avons donc effectué une recherche d'IgE vis-à-vis de Pru p 7 pour ces patients [8].

Pru p 7 ou peamacléine est un peptide antimicrobien de la famille des gibberellin regulated protein [9-12]. Ces protéines interviennent dans la défense des végétaux et plus particulièrement des fruits avec une fonction antimicrobienne. On les trouve aussi bien dans la peau que dans la pulpe du fruit. Des homologues sont décrits dans divers aliments d'origine végétale : la pêche, l'orange (Cit s 7) [13], la grenade (Pun g 7 : pommaclein) [11], l'abricot du Japon (Pru m 7), ainsi que dans le tubercule de pomme de terre [9].

La peamacléine est une petite molécule de $7 \mathrm{kDa}$ (par comparaison, la LTP Pru p 3 a une masse moléculaire de $9 \mathrm{kDa}$ ) comprenant 63 acides aminés. Elle est riche en cystéines avec $19 \%$ de résidus cystéine et 6 ponts disulfure lui conférant une structure mixte : $\alpha$-hélicoïdale et feuillet $\beta$-plissé.

La peamacléine est une protéine basique avec un point isoélectrique situé à 8,97, proche de celui de Pru p 3 à 9,25. Elle est thermorésistante et résistante à la digestion acide, par contre, elle n'est pas digérée par la trypsine, contrairement à Pru p 3 qui l'est partiellement.

De masse moléculaire et point isoélectrique proches, Pru $\mathrm{p}$ 3 et Pru p 7 sont difficilement séparables. Il est donc possible d'avoir une contamination de nPru p 3 par Pru p 7.

Afin d'évaluer la fréquence de sensibilisation vis-à-vis de ce nouvel allergène dans la région de Marseille, nous avons sélectionné des sérums de patients pour lesquels une histoire clinique d'allergie à la pêche avait été évoquée, ainsi que des patients avec une histoire d'anaphylaxie sans cause retrouvée malgré une anamnèse minutieuse et des tests cutanés et biologiques exhaustifs.

\section{Patients et méthodes}

\subsection{Patients}

Étude rétrospective monocentrique en soin courant des dossiers et des résultats biologiques de 121 patients explorés pour une suspicion d'allergie à la pêche dans le réseau ANAFORCAL Provence et au laboratoire d'immunologie de l'hôpital de La Conception ou dans un laboratoire privé du réseau SELDAIXBIOPLUS, entre février 2012 et juin 2016.

Recueil des arguments diagnostiques : histoire clinique, résultats des tests cutanés avec l'extrait commercial et/ou l'aliment natif, sensibilisation biologique vis-à-vis des extraits 
et des allergènes moléculaires disponibles pour le diagnostic in vitro.

\subsection{Corrélation entre les données cliniques et biologiques}

Les données cliniques relatives aux symptômes d'allergie alimentaire et le résultat des TC à lecture immédiate ont été comparés aux données biologiques.

La gravité de la réaction immédiate clinique a été classée selon les 3 grades de sévérité d'anaphylaxie décrits par Muraro, adapté de Sampson $[14,15]$.

Ces auteurs retiennent le diagnostic d'anaphylaxie comme hautement probable si 1 des 3 critères suivants est rempli :

- réaction aiguë (minutes à quelques heures) à type de signes cutanéo-muqueux (urticaire généralisée, prurit, bouffées vaso-motrices, œdème de la langue et luette) associés à au moins 1 des éléments suivants : signes respiratoires (dyspnée, bronchospasme, stridor, hypoxie) ou signes cardiovasculaires à type d'hypotension ;

- au moins 2 ou plus des critères suivants apparaissant dans les minutes à quelques heures après exposition à un allergène probable pour ce patient : signes cutanéo-muqueux (urticaire généralisée, prurit, bouffées vaso-motrices, œdème de la langue et luette), respiratoires (dyspnée, bronchospasme, stridor, hypoxie), cardiovasculaires (hypotension) et/ou signes digestifs persistants (douleurs abdominales, vomissements) ;

- état de choc : hypotension de plus de $30 \%$ par rapport à la tension artérielle basale, collapsus, après exposition à un allergène connu du patient, survenant dans les minutes à quelques heures.

Notons que la définition des auteurs «minutes à quelques heures » peut être rapprochée de l'intervalle généralement accepté de 4 heures maximum.

\subsection{Prélèvements}

Les prélèvements veineux ont été réalisés sur tube sans anticoagulant, sans conditions pré-analytiques particulières (le résultat du dosage d'IgE spécifiques est connu pour être indépendant d'une prise médicamenteuse ou alimentaire). Les tubes ont été centrifugés et le sérum conservé à $+4{ }^{\circ} \mathrm{C}$ pendant une semaine, $-20^{\circ} \mathrm{C}$ au-delà jusqu'à réalisation du dosage d' $\operatorname{IgE}$ spécifiques.

Les TAB ont été effectués sur sang total dans les 2 heures suivant le prélèvement veineux sur tube EDTA.

\subsection{Méthodes, appareils et réactifs}

\subsubsection{IgE spécifiques unitaires}

La recherche et le dosage des IgE spécifiques ont été réalisés chez les 121 patients par la méthode fluoro-immunoenzymatique FEIA ImmunoCAP ${ }^{\circledR}$ de Thermo Fisher (ImmunoCAP 250, Thermo Fisher, Uppsala, Suède). Cette méthode nécessite une prise d'essai de $40 \mu \mathrm{L}$ de sérum (volume mort $+20 \mu \mathrm{L}$ ). C'est une méthode quantitative avec un rendu de résultats linéaire entre $0,10 \mathrm{kUA} / \mathrm{L}$ et $100 \mathrm{kUA} / \mathrm{L}$. Le seuil de positivité a été fixé à 0,10 kUA/L [16-19].

Les dosages d'IgE spécifiques vis-à-vis de l'extrait de pêche, des allergènes moléculaires Pru p 1, Pru p 3, Pru p 4 et CCD (groupements MUXF3, o214) et du pollen de Cupressacées (extrait de pollen de cyprès $t 23$, de genévrier t6 ou allergène majeur et spécifique nCup a 1 t226) ont été réalisés avec les réactifs commercialisés pour diagnostic in vitro, selon des méthodes accréditées COFRAC.

La recherche et le dosage d'IgE spécifiques vis-à-vis de Pru p 7 ont été effectués par le département R\&D de Thermo Fisher ImmunoDiagnostics (Uppsala, Suède), en utilisant Pru p 7 natif et recombinant en couplage expérimental pour FEIA ImmunoCAP.

Chez 6 patients, la recherche d'IgE spécifiques vis-à-vis des allergènes moléculaires Pru p 1, Pru p 3 et Pru p 4 sous forme purifiée a été contrôlée par méthode Immulite (Siemens, Munich, Italie).

\subsubsection{Multitest FABER ${ }^{\circledR}$}

Une exploration par multitest FABER (CAAM, Rome, Italie) a été réalisée chez 6 patients. La méthode FABER offre un panel mixte de 122 extraits allergéniques et 122 allergènes moléculaires, incluant deux extraits de pêche (pulpe et peau), les allergènes Pru $p$ 1, Pru $p 3$ et Pru p 7, ainsi que l'homologue Pun g 7 (grenade). FABER est une méthode semi-quantitative, avec un rendu de résultat en unités qui lui sont propres (fluorescence intensity units [FIU]).

\subsubsection{Tests d'activation des basophiles (TAB)}

Un TAB (trousse et réactifs de Bühlmann Laboratories, Suisse) en présence d'extrait de pêche et des allergènes moléculaires nPru p 3 (LTP), Mal d 1 (réactif PR-10), Tri a 12 (réactif profiline) a été réalisé pour 10 patients suivant une procédure standard avec 4 concentrations par allergène (concentrations finales 62,5 ng/mL, 31,3 ng/mL, 12,5 ng/mL et $1,25 \mathrm{ng} / \mathrm{mL}$ ). L'extrait de lait de vache a servi ici comme témoin de bonne tolérance clinique (tous les patients consommaient du lait et des produits laitiers régulièrement et sans problème).

Dans un deuxième temps, un TAB en présence de Pru $p$ 7 et de Pru p 3 purifiés et recombinants (fournis gracieusement par Thermo Fisher) a été réalisé pour 7 patients. Pour cette seconde série de $\mathrm{TAB}, 11$ concentrations de chaque allergène ont été testées avec deux méthodes : le kit commercial Bühlmann Laboratories et une méthode Beckman-Coulter. Le TAB a été considéré positif quand le pourcentage de basophiles exposés à l'allergène et exprimant CD63 était au moins deux fois supérieur au témoin négatif. Nous avons testé 7 patients, dont 6 allergiques sévères à la pêche et présentant des IgE Pru p 7 isolées (sans IgE Pru p 1, Pru p 4, Pru p 3 ou CCD) et un patient présentant des épisodes anaphylactiques répétés avec des aliments végétaux, sans IgE Pru p 1, Pru p 3, Pru p 4, Pru p 7 ou CCD. 


\subsection{Expression des résultats et analyse statistique}

Les données sont exprimées en médiane et extrêmes. L'analyse statistique a été effectuée avec le test de MannWhitney et coefficient de détermination $\mathrm{R}^{2}$. Les résultats sont considérés comme statistiquement significatifs pour $p \leq 0,05$.

\section{Résultats}

\subsection{Démographie et diagnostic retenu}

Parmi les 121 patients, on trouve 51 hommes (sex-ratio homme femme : 0,73). L'âge médian est de 19,8 ans (4-74).

L'analyse des données cliniques (histoire clinique et résultats des tests cutanés) a permis de distinguer 3 groupes de patients : allergiques confirmés à la pêche $(n=74)$, non allergiques (tolérants) à la pêche $(n=35)$ et impossibles à conclure. En effet, pour 12 patients, le diagnostic d'allergie alimentaire à la pêche n'a pas pu être établi (renseignements cliniques insuffisants, tests cutanés ininterprétables ou manquants, histoire clinique peu convaincante).

\subsection{Caractérisation de la sensibilisation vis-à-vis de Pru p} 7

Sur les 121 sérums analysés, $112(93 \%)$ se sont révélés positifs vis-à-vis de l'extrait de pêche et $77(64 \%)$ ont montré une réactivité IgE vis-à-vis de rPru p 7. Parmi les 74 patients allergiques confirmés à la pêche, 58 (78\%) avaient des IgE anti-Pru p 7 positives (Tableau 1).

Dans 49/77 (64 \%) des cas, la réactivité IgE à Pru p 7 était isolée (pas de détection d'IgE vis-à-vis de rPru p 1, rPru p 3 ou rPru p 4).
Tableau 1

Profil moléculaire de la pêche dans les 3 groupes de patients : allergiques à la pêche $(n=74)$, tolérants à la pêche $(n=35)$ et patients non groupables $(n=12)$.

\begin{tabular}{lllll}
\hline Prévalence & Pru p 1 & Pru p 3 & Pru p 4 & Pru p 7 \\
\hline $\begin{array}{l}\text { Allergiques à la pêche, } \\
\quad 74 \text { patients }\end{array}$ & $15(20 \%)$ & $21(28 \%)$ & $12(16 \%)$ & $58(78 \%)$ \\
$\begin{array}{l}\text { Tolérants à la pêche, } \\
\quad 35 \text { patients }\end{array}$ & $7(20 \%)$ & $14(40 \%)$ & $12(34 \%)$ & $11(31 \%)$ \\
$\begin{array}{l}\text { Renseignements cliniques } \\
\text { insuffisants ou non } \\
\text { obtenus, 12 patients }\end{array}$ & $5(42 \%)$ & $2(17 \%)$ & $3(25 \%)$ & $8(67 \%)$ \\
\hline
\end{tabular}

Les taux d'IgE spécifiques vis-à-vis de Pru p 7 étaient plus élevés que ceux vis-à-vis de l'extrait de pêche (médiane : 4,9 contre $1,3 \mathrm{kUA} / \mathrm{L}$; respectivement intervalles : 0,10 à 30,72 ; $0,10$ à 52,$4 ; p=0,003$; coefficient de corrélation $-0,15)$ (Fig. 1).

Parmi les 7 sérums négatifs en IgE contre la pêche, deux ont été trouvés positifs vis-à-vis de rPru p 7 (0,81 et 1,24kUA/L). De même, parmi les 19 sérums pour lesquels les valeurs d' IgE contre la pêche étaient comprises entre 0,10 et $0,35 \mathrm{kUA} / \mathrm{L}, 14$ ont été trouvés positifs à rPru p $7(0,10$ à $6,44 \mathrm{kUA} / \mathrm{L})$ avec des valeurs d'IgE supérieures à celles obtenues avec l'extrait.

Tous les patients sensibilisés à Pru p 7 étaient sensibilisés aux pollens de Cupressacées, mais l'inverse n'était pas vrai.

\subsection{Profil moléculaire de sensibilisation et particularités cliniques}

Parmi les 74 patients allergiques confirmés à la pêche, 41 (55\%) décrivent une réaction clinique sévère grade III après ingestion de pêche. Au total, 24 patients (32\%) décrivent l'existence d'un cofacteur, le plus souvent l'effort (17 patients sur 24), parfois la prise d'alcool. Une particularité clinique à type

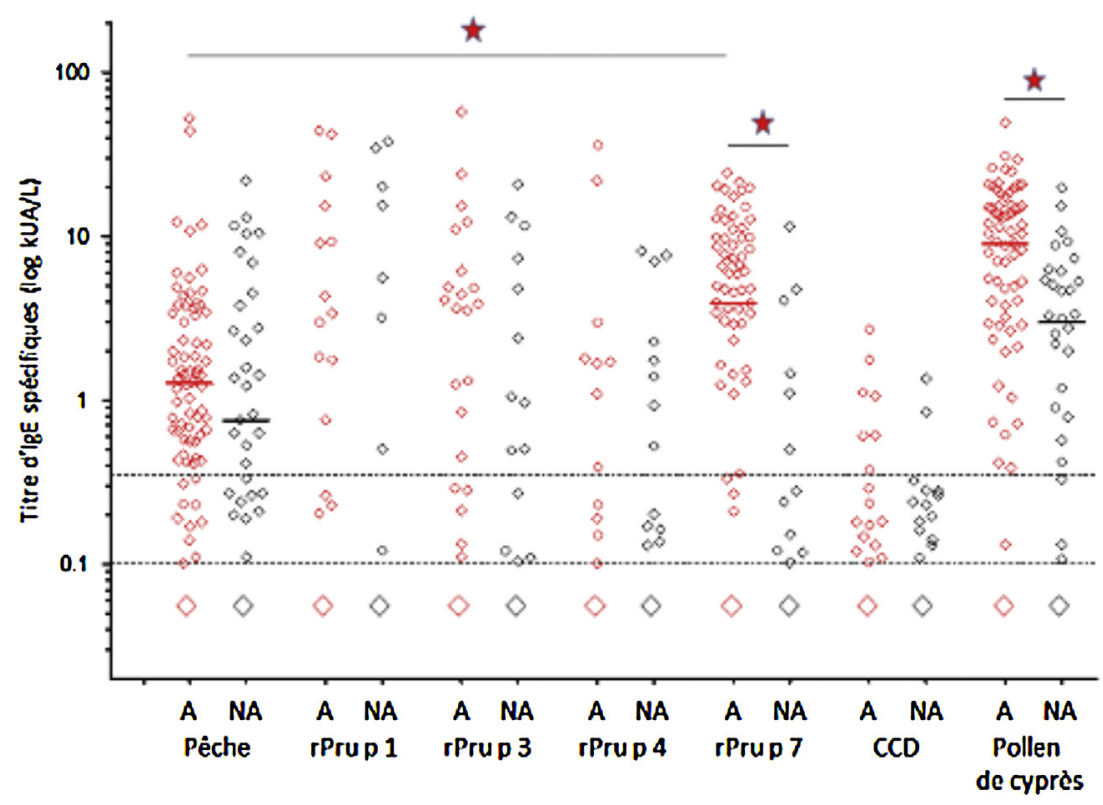

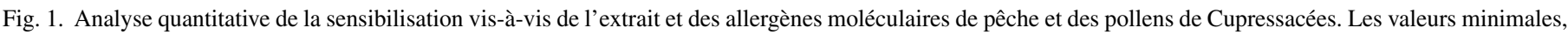

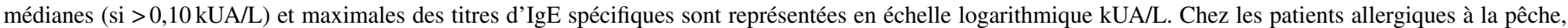

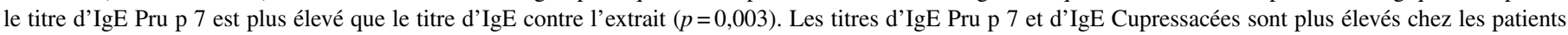
allergiques à la pêche $(p<0,01)$. Il n'y a pas de différence significative pour les IgE Pru p 1, Pru p 3 et Pru p 4. 
Tableau 2

Prévalence de la sensibilisation biologique à Pru p 7 et données cliniques dans les 2 populations de patients : allergiques à la pêche ( $n=74)$ et tolérants à la pêche $(n=35)$.

\begin{tabular}{|c|c|c|c|c|c|c|c|}
\hline & $\begin{array}{l}\text { Prévalence de la } \\
\text { sensibilisation à } \\
\text { Pru p } 7 \text { (effectif et } \\
\% \text { ) }\end{array}$ & $\begin{array}{l}\text { Anaphylaxie } \\
\text { grade I : } \\
\text { MURARO }\end{array}$ & $\begin{array}{l}\text { Anaphylaxie } \\
\text { grade II : } \\
\text { MURARO }\end{array}$ & $\begin{array}{l}\text { Anaphylaxie } \\
\text { grade III : } \\
\text { MURARO }\end{array}$ & $\begin{array}{l}\text { Gravité de la } \\
\text { réaction non } \\
\text { précisée }\end{array}$ & $\begin{array}{l}\text { Cofacteur } \\
\text { identifié }\end{array}$ & $\begin{array}{l}\text { Edème de } \\
\text { la face }\end{array}$ \\
\hline $\begin{array}{l}\text { Allergiques à la } \\
\text { pêche, } 74 \text { patients }\end{array}$ & $58(78 \%)$ & $17(23 \%)$ & $10(14 \%)$ & $41(55 \%)$ & $6(8 \%)$ & $24(32 \%)$ & $39(53 \%)$ \\
\hline $\begin{array}{l}\text { Tolérants à la pêche, } \\
35 \text { patients }\end{array}$ & $11(31 \%)$ & $14(40 \%)$ & $3(9 \%)$ & $7(20 \%)$ & $11(31 \%)$ & $1(3 \%)$ & $5(14 \%)$ \\
\hline
\end{tabular}

Pour 6 patients dans le groupe allergiques, et 11 patients dans le groupe tolérants, l'analyse des renseignements cliniques n'a pas permis de classer la gravité de la réaction allergique.

d'œdème de la face est retrouvée chez 39 (53 \%) des patients allergiques confirmés à la pêche (Tableau 2).

Chez les patients allergiques à la pêche, des différences cliniques sont observées selon le profil moléculaire de sensibilisation (Tableau 3). Les réactions cliniques sévères, l'existence d'un œdème de la face et la présence de cofacteurs sont plus fréquents chez les patients avec sensibilisation biologique vis-à-vis de Prup 7.

En fonction de l'allergène moléculaire détecté, d'autres particularités cliniques ont été notées. Les 3 grades de sévérité clinique sont possibles lorsque des IgE contre la LTP Pru p 3 sont en cause. Les seules réactions cliniques grade III associées à un profil PR-10 Pru p 1 ou profiline Pru p 4 sont survenues chez des patients co-sensibilisés avec Pru p 7.

\subsection{Multitest FABER}

La présence de Pru $\mathrm{p} 7$ et de son homologue de la grenade Pun $\mathrm{g} 7$ a justifié la réalisation d'une exploration FABER, confirmant la positivité Pru p 7 chez 5 patients (5/6).

Sur le multitest FABER, trois patients étaient également positifs pour l'extrait de pêche : 2 vis-à-vis de la peau uniquement, 1 vis-à-vis de la peau et la pulpe. La négativité vis-à-vis de Pru p 3 a été confirmée en FABER pour les 6 patients.

Pour un patient, les IgE spécifiques unitaires et le TAB se sont révélés positifs pour Pru p 7 malgré un résultat FABER négatif.

\subsection{Exploration de la capacité activatrice de Pru 7 en test fonctionnel : test d'activation des basophiles}

\subsubsection{TAB avec extrait de pêche et nPru $\mathrm{p} 3$ Bühlmann Laboratories}

Les TAB réalisés en présence d'extrait de pêche et de nPru p 3 pur à $95 \%$ (Bühlmann Laboratories) étaient positifs chez 10/10 patients pour l'extrait de pêche et pour nPru p 3. Ils étaient négatifs pour Mal d 1 (réactif représentant la famille PR-10), pour Tri a 12 (réactif représentant la famille des profilines) et pour l'extrait de lait de vache servant ici de témoin de bonne tolérance clinique.

\subsubsection{TAB avec nPru $p$ 7, rPru $p$ 7, nPru $p 3$ et rPru $p$ 3 Thermo Fisher}

Les TAB réalisés en présence de nPru $\mathrm{p} 7$ et de rPru $\mathrm{p} 7$ étaient positifs chez les 6 patients avec des IgE unitaires contre Pru $p$ 7 détectables (6/6) et négatif chez le patient sans IgE détectées contre Pru p 7, prouvant l'activité allergénique de Pru p 7 et la spécificité de la réaction. L'ensemble des TAB était négatif avec les allergènes $r$ Pru $\mathrm{p} 3$ et nPru $\mathrm{p} 3$ de pureté vérifiée par spectrométrie de masse pour l'ensemble de la gamme de concentrations finales testées, allant de $0,25 \mathrm{pg} / \mathrm{mL}$ à $2,5 \mu \mathrm{g} / \mathrm{mL}$.

La concordance $\mathrm{TAB}$-multitest FABER pour la positivité Pru p 7 était de 5/6, le patient sans IgE Pru p 7 détectables par méthode FABER réagissant fortement à nPru $\mathrm{p} 7$ et rPru $\mathrm{p} 7$ lors du TAB (mais avec des IgE unitaires positives contre Pru p 7).

Les allergènes nPru $\mathrm{p} 7$ et rPru $\mathrm{p} 7$ ont démontré au cours des $\mathrm{TAB}$ la capacité à activer les basophiles dès la concentration de

Tableau 3

Particularités cliniques en fonction du profil de sensibilisation moléculaire chez les patients allergiques confirmés vis-à-vis de la pêche $(n=74)$.

\begin{tabular}{|c|c|c|c|c|}
\hline $\begin{array}{l}\text { Particularités cliniques et } \\
\text { cofacteurs (nombre de } \\
\text { patients) }\end{array}$ & Pru p 1 & Pru p 3 & Pru p 4 & Pru p 7 \\
\hline MURARO I & 3 (dont 1 co-sensibilisé Pru p 7) & 7 (dont 4 co-sensibilisés Pru p 7) & 3 (dont 1 co-sensibilisé Pru p 7) & 10 \\
\hline MURARO III & 1 (co-sensibilisé Pru p 7) & 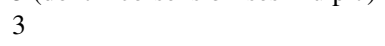 & 1 (co-sensibilisé Pru p 7) & 37 \\
\hline Edème de la face & 6 (dont 4 co-sensibilisés Pru p 7) & 6 (dont 2 co-sensibilisés Pru p 7) & 1 & 31 \\
\hline Cofacteurs & 3 (dont 2 co-sensibilisés Pru p 7) & 1 & 0 & 21 \\
\hline
\end{tabular}

Pour 4 patients sensibilisés à Pru p 7, l'analyse du recueil des renseignements cliniques n'a pas permis de classer la gravité de la réaction. Pour un patient avec cofacteur et œdème de la face, aucun substrat moléculaire de la pêche n'a été mis en évidence. 


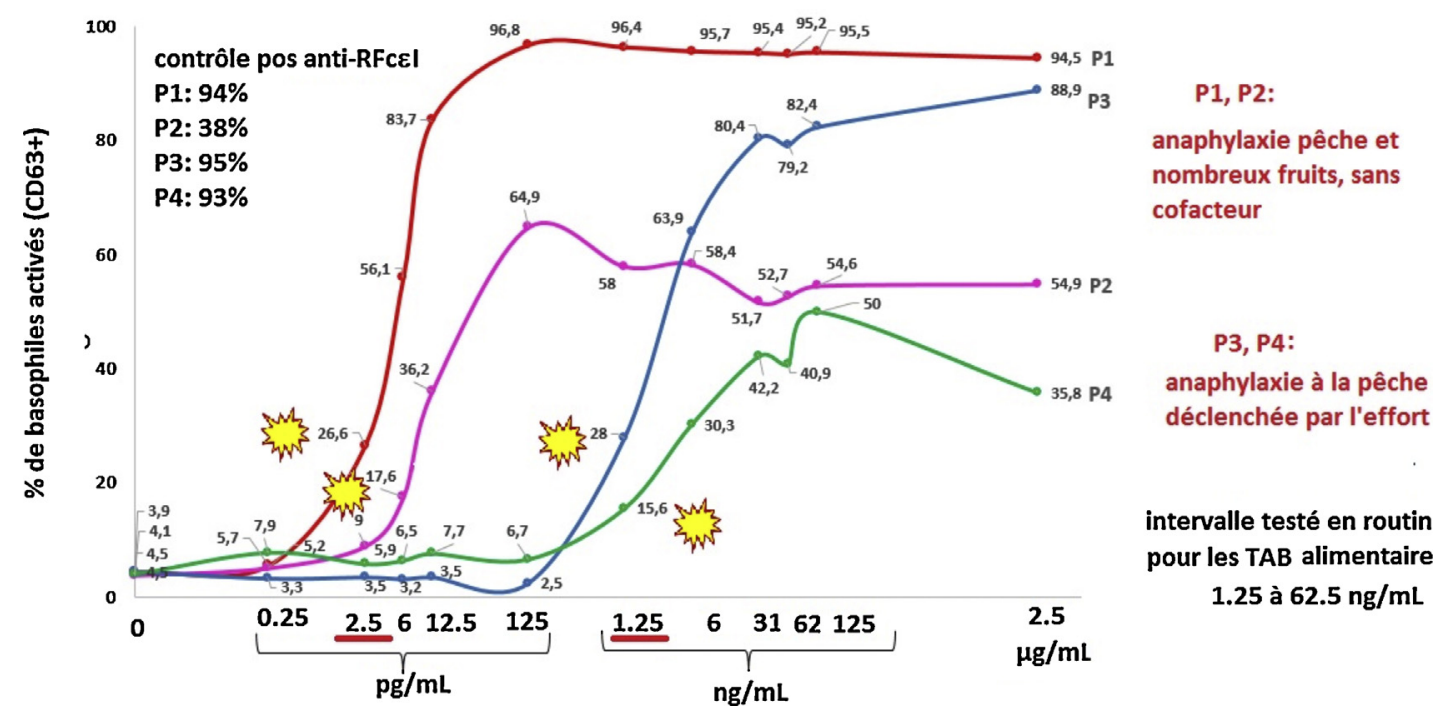

Fig. 2. Courbes dose-réponse de l'activation des basophiles avec Pru p 7 chez 4 patients représentatifs. La concentration minimale activatrice est indiquée pour chaque patient par une étoile. L'intensité maximale d'activation en présence de Pru p 7 peut être comparée à l'intensité observée avec le contrôle positif anti-RFc epsilon I pour chaque patient. On observe deux profils de réponse : dose activatrice de $2,5 \mathrm{pg} / \mathrm{mL}$ de Pru p 7 pour les basophiles des patients réagissant sans cofacteur et de $1,25 \mathrm{ng} / \mathrm{mL}$ pour les basophiles des patients réagissant avec un cofacteur. L'intensité maximale d'activation avec Pru p 7 est comparable avec l'intensité d'activation avec le contrôle positif, sauf pour un patient (P4) chez qui elle reste inférieure d'environ $50 \%$.

$2,5 \mathrm{pg} / \mathrm{mL}(5 / 6$ patients), $500 \times$ inférieure à la dernière concentration de la gamme que nous utilisons en routine $(1,25 \mathrm{ng} / \mathrm{mL})$ et $10000 \times$ inférieure à la concentration habituellement recommandée pour un aliment, à savoir 25 à $100 \mathrm{ng} / \mathrm{mL}$ (Fig. 2).

\section{Discussion}

L'analyse de 121 patients de la région de Marseille a permis de montrer et décrire l'implication de la peamacléine (Pru p 7) chez $78 \%$ des patients allergiques confirmés à la pêche versus $28 \%$ pour Pru p 3, $20 \%$ pour Pru p 1 et $16 \%$ pour Pru p 4 . Pru p 7 est dans notre série le seul allergène majeur chez ces patients. La pertinence fonctionnelle de Pru p 7 est confirmée par la positivité des tests d'activation des basophiles chez les patients présentant des IgE vis-à-vis de Pru p 7. Un élément remarquable est l'activation des basophiles dès la zone des très faibles doses de Pru p 7 (2,5 pg/mL). La sensibilisation vis-à-vis de Pru p 7 est présente dès l'enfance, avec un âge minimum de 4 ans et une médiane autour de 20 ans.

Parmi les raisons pouvant expliquer l'absence de description de cette sensibilisation à forte prévalence il nous semble important de souligner les mauvaises performances des extraits de pêche, souvent utilisés pour le diagnostic de première intention. Dans notre population, de très faibles valeurs d' $\operatorname{IgE}$ unitaires visà-vis de l'extrait de pêche (indétectables ou 0,10-0,35 kUA/L) étaient associées à des IgE contre Pru p 7 allant jusqu'à 6,44 kUA/L. Le titre d'IgE vis-à-vis de Pru p 7 était systématiquement supérieur au titre d'IgE contre l'extrait de pêche chez les 77 patients sensibilisés à Pru p 7. De manière similaire, des IgE contre les extraits de pêche (peau et pulpe) présents sur le multitest FABER étaient détectées seulement chez 2 patients parmi les 5 présentant des IgE Pru $\mathrm{p} 7$ avec cette même méthode. Pris ensemble, ces résultats suggèrent que Pru $p 7$ est insuffisamment représenté dans les extraits utilisés pour les réactifs de diagnostic IgE in vitro. Une évaluation des extraits commerciaux pour tests cutanés pourrait être utile, bien que l'utilisation des aliments natifs reste la référence dans ce domaine.

Sur le plan clinique, une étude d'Inomata et al. de 2014 a montré que la peamacléine était un marqueur de sévérité clinique dans l'allergie alimentaire à la pêche au Japon [20]. De manière similaire, dans notre population d'étude, Pru p 7 est associé à des réactions cliniques sévères : les IgE vis-à-vis de Pru $\mathrm{p} 7$ sont présentes chez 37 des 41 patients allergiques à la pêche et classés grade III de Muraro [14].

Dans notre étude, l'intervention d'un cofacteur, généralement l'effort physique, est très fréquente en association avec la sensibilisation à Pru $\mathrm{p} 7$ (21 patients/24). Ce résultat pourrait expliquer l'échec fréquent dans l'identification d'un substrat moléculaire dans les anaphylaxies alimentaires aux fruits induites par l'effort. Dans la littérature, on retrouve des cas d'anaphylaxie liée aux fruits des Rosacées et induite par l'effort, parfois attribuée au substrat LTP dans les régions méditerranéennes [21]. Dans d'autres cas, le substrat moléculaire reste inconnu, notamment pour les réactions à la pêche. L'implication d'IgE vis-à-vis d'une isoforme de Pru p 3 différente de celle utilisée pour la recherche d'IgE spécifiques en routine et donc non détectables par les méthodes actuelles a été proposée [22-24]. Compte tenu de la parenté physico-chimique de Pru p 3 et de Pru $\mathrm{p} 7$ et de la capacité activatrice de Pru $\mathrm{p} 7$ à des doses très inférieures à celles habituellement observées avec les allergènes alimentaires, du moins in vitro, la présence de Pru p 7 dans les préparations utilisées pour certaines études antérieures ne peut être exclue.

Une autre particularité clinique des patients allergiques à la pêche et sensibilisés à Pru p 7 est la survenue fréquente d'un œdème de la face, rapportée chez les patients japonais [25] et confirmée dans notre étude avec une prévalence de 31/58 $(53 \%)$. 
Enfin, nous avons également documenté la présence constante d'IgE anti-Cupressacées chez les patients sensibilisés à Pru $\mathrm{p}$ 7. Chez chaque patient, le titre d'IgE vis-à-vis du pollen de Cupressacées est systématiquement supérieur au titre d'IgE vis-à-vis de Pru p 7. Cette étude ayant été réalisée uniquement chez des patients de la région de Marseille, il serait intéressant de la compléter par une étude sur la sensibilisation à Pru p 7 hors de la zone de pollinisation des Cupressacées afin de mieux comprendre les liens entre Pru p 7 et les pollinoses.

\section{Conclusion}

Pru p 7 complète le profil moléculaire de la pêche et s'avère nécessaire pour résoudre le diagnostic d'allergie à la pêche chez les patients méditerranéens. Dans l'allergie à la pêche, la mise en évidence d'un substrat moléculaire Pru p 7 est un facteur pronostique de sévérité clinique, d'anaphylaxie alimentaire à la pêche induite par l'exercice et d'une présentation clinique incluant fréquemment un œdème de la face.

La cosensibilisation constante au pollen de Cupressacées place Pru p 7 en position de candidat pour le chaînon manquant du syndrome cyprès-pêche des régions méditerranéennes.

\section{Déclaration de liens d'intérêts}

Jonas Lidholm, Angelica Ehrenberg et Jonas Östling sont employés par Thermo Fisher. Rihane Arif-Lusson est doctorante en thèse CIFRE en collaboration avec Beckman Coulter. Les autres auteurs déclarent ne pas avoir de liens d'intérêts.

\section{Remerciements}

Mme Colette Mavier et Mme Catherine Tissot (Thermo Fisher), Dr Fabrice Guerber (Laboratoires Oriade-Noviale, Grenoble).

\section{Références}

[1] Werfel T, Asero R, Ballmer-Weber BK, Beyer K, Enrique E, Knulst $\mathrm{AC}$, et al. Position paper of the EAACI: food allergy due to immunological cross-reactions with common inhalant allergens. Allergy 2015;70:1079-90.

[2] Popescu FD. Cross-reactivity between aeroallergens and food allergens. World J Methodol 2015;5:31-50.

[3] Mastrorilli C, Tripodi S, Caffarelli C, Perna S, Di Rienza-Businco A, Sfika I, et al. Endotypes of pollen-food syndrome in children with seasonal allergic rhinoconjunctivitis: a molecular classification. Allergy 2016:1181-91.

[4] Andersen MB, Hall S, Dragsted LO. Identification of european allergy patterns to the allergen families PR-10, LTP, and profilin from Rosaceae fruits. Clin Rev Allergy Immunol 2011;41:4-19.

[5] Tenenbaum J, Leoni MC, Demoly MP, Bourrain JL, Demoly P, Chiriac AM. Allergie à la pêche avec taux d'IgE spécifiques pêche et allergènes majeurs négatifs ou faiblement positifs. Rev Fr Allergol 2016;56:441-5.

[6] Klingebiel C, Poisson A, Rousseau M, Guieu C, Cleach I, Mège JL, et al. Sensibilisation Pru p 3 chez l'allergique à la pêche : apport du test d'activation des basophiles chez les patients avec IgE Pru p 3 indétectables. Rev Fr Allergol 2016;56:282.

[7] Klingebiel C, Poisson A, Rousseau M, Guieu C, Cleach I, Mège J-L, et al. The basophil activation test confirms Pru p 3 sensitization in peach-allergic patients with negative sIgE to Pru p 3. Allergy 2016;71(S102):154.

[8] Klingebiel C, Lidholm J, Ehrenberg A, Östling J, Cleach I, Mège JL, et al. Pru p 7 is a major peach allergen in patients from Southern France. Clin Transl Allergy 2017;7(S1):OP18.

[9] Tuppo L, Alessandri C, Pomponi D, Picone D, Tamburrini M, Ferrara $\mathrm{R}$, et al. Peamaclein - a new peach allergenic protein: similarities, differences and misleading features compared to Pru p 3. Clin Exp Allergy 2013;43:128-40.

[10] Tuppo L, Spadaccini R, Alessandri C, Wienk H, Boelens R, Giangrieco I, et al. Structure, stability, and IgE binding of the peach allergen peamaclein (Pru p 7). Biopolymers (Peptide Science) 2014;102:416-25.

[11] Tuppo L, et al. Pomegranate cultivars: identification of the new IgE-binding protein pommaclein and analysis of anti-oxidant variability. J Agric Food Chem 2017;65(13):2702-10.

[12] Porto WF, Franco OL. Theoretical structural insights into the snakin/GASA family. Peptides 2013;44:163-7.

[13] Inomata N, Miyagawa M, Aihara M. Identification of orange peamaclein as a new allergen in orange allergy. Allergy 2015;70(S102):107.

[14] Muraro A, Roberts G, Clark A, Eigenmann PA, Halken S, Lack G, et al. The management of anaphylaxis in childhood: position paper of the European academy of allergology and clinical immunology. Allergy 2007;62:857-71.

[15] Sampson HA, Munoz-Furlong A, Campbell RL, Adkinson Jr NF, Bock SA Branum A, et al. Second symposium on the definition and management of anaphylaxis: summary report — second national institute of allergy and infectious disease/food allergy and anaphylaxis network symposium. J Allergy Clin Immunol 2006;117:391-7.

[16] Kleine-Tebbe J, Poulsen LK, Hamilton RG. Quality management in IgEbased allergy diagnostics. J Lab Med 2016;40:81-96.

[17] Lambert C, Sarrat A, Bienvenu F, Brabant S, Nicaise-Roland P, Alyanakian MA, et al. The importance of EN ISO 15189 accreditation of allergenspecific IgE determination for reliable in vitro allergy diagnosis. Allergy 2015;70:180-6.

[18] Hamilton RG. Clinical laboratories worldwide need to report IgE antibody results on clinical specimens as analytical results and not use differential positive thresholds. J Allergy Clin Immunol 2015;136:811-2.

[19] Hamilton RG. Allergic sensitization is a key risk factor for but not synonymous with allergic disease. J Allergy Clin Immunol 2014;134:360-1.

[20] Inomata N, Okazaki F, Moriyama T, Nomura Y, Yamaguchi Y, Honjoh $\mathrm{T}$, et al. Identification of peamaclein as a marker allergen related to systemic reactions in peach allergy. Ann Allergy Asthma Immunol 2014; 112:175-83.

[21] Romano A, Scala E, Rumi G, Gaeta F, Caruso C, Alonzi C, et al Lipid transfer proteins: the most frequent sensitizer in Italian subjects with food-dependent exercise-induced anaphylaxis. Clin Exp Allergy 2012;42:1643-53.

[22] Bianchi A, Di Rienzo Businco A, Bondanini F, Mistrello G, Carlucci A, Tripodi S. Rosaceae-associated exercise-induced anaphylaxis with positive SPT and negative IgE reactivity to Pru p 3. Eur Ann Allergy Clin Immunol 2011;43:122-4

[23] Miceli Sopo S, Monaco S, Giorgio V, Calvani M, Mistrello G, Onesimo R. Food-dependent exercise-induced anaphylaxis (FDEIA) by nectarine in a paediatric patient with weakly positive nectarine prick-by-prick and negative specific IgE to Pru p 3. Allergol Immunopathol 2013;41:201-3.

[24] Hotta A, Inomata N, Tanegasima T, Oda K, Inoue Y, Aihara M. Case of food-dependent exercise-induced anaphylaxis due to peach with Pru p 7 sensitization. J Dermatol 2016;43:222-3.

[25] Inomata N, Miyakawa M, Aihara M. Eyelid edema as a predictive factor for sensitization to Pru p 7 in peach allergy. J Dermatology 2016;43:900-5. 